\title{
Isocitrate dehydrogenase inhibitors in acute myeloid leukemia
}

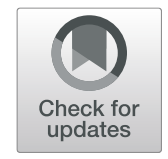

\author{
Xiaoyan Liu and Yuping Gong ${ }^{*}$ (D)
}

\begin{abstract}
Isocitrate dehydrogenase (IDH) is a key enzyme involved in the conversion of isocitrate to a-ketoglutarate (a-KG) in the tricarboxylic acid (TCA) cycle. IDH mutation produces a neomorphic enzyme, which can lead to the abnormal accumulation of R-2-HG and promotes leukemogenesis. IDH mutation occurs in $20 \%$ of acute myeloid leukemia (AML) patients, mainly including IDH1 R132, IDH2 R140, and IDH2 R172. Different mutant isoforms have different prognostic values. In recent years, IDH inhibitors have shown good clinical response in AML patients. Hence, enasidenib and ivosidenib, the IDH2 and IDH1 inhibitors developed by Agios Pharmaceuticals, have been approved by the Food and Drug Administration on 1 August 2017 and 20 July 2018 for the treatment of adult relapsed or refractory (R/R) AML with IDH2 and IDH1 mutations, respectively. IDH inhibitor monotherapy for R/R AML is efficacious and safe; however, there are problems, such as primary or acquired resistance. Clinical trials of IDH inhibitors combined with hypomethylating agents or standard chemotherapy for the treatment of R/R AML or newly diagnosed AML, as well as in post hematopoietic stem cell transplantation as maintenance therapy, are ongoing. This article summarizes the use of IDH inhibitors in AML with IDH mutations.
\end{abstract}

Keywords: AML, IDH mutation, IDH inhibitor

\section{Introduction}

Approximately $40-45 \%$ of young and $10-20 \%$ of elderly AML patients treated with standard therapy will be cured. There are no more than $10 \%$ of relapsed or refractory $(\mathrm{R} /$ R) AML [1-4]. Advancements in next-generation sequencing (NGS) have increased our understanding on genomic and epigenomic landscapes in AML [5, 6], and therefore, precision therapy has become possible in AML treatment, multiple studies of targeted drugs (such as IDH inhibitors, FLT3 inhibitors and BCL-2 inhibitors, etc.) are ongoing [7-12]. Isocitrate dehydrogenase (IDH), converts isocitrate to $\alpha$-ketoglutarate $(\alpha-K G)$ and has three different isoforms. IDH1 and IDH2 are located in the cytoplasm and mitochondria, respectively, while IDH3 is located in the mitochondrial matrix [13]. Recurring IDH1 and IDH2 mutations were reported in glioma in 2008 and 2009, respectively $[14,15]$, and later in AML, cholangiocarcinoma, chondrosarcoma, chondromas, and so on [16-18]. To date, no study has reported cancer-associated mutations

\footnotetext{
* Correspondence: gongyuping2010@aliyun.com Department of Hematology, West China Hospital of Sichuan University, No.37 Guo Xue Xiang, Chengdu 610041, Sichuan Province, China
}

in IDH3, although IDH3 has a similar function as that of IDH1 and IDH2.

IDH mutations occur in the active catalytic sites of the arginine residues resulting in a neomorphic enzyme activity converting $\alpha$-KG to the oncometabolite R-2-hydroxygluta rate (R-2-HG) [19, 20]. The abnormal accumulation of R-2-HG affects multiple $\alpha$-KG-dependent dioxygenases including abnormal methylation of histones and DNA [21-24], blocking cell differentiation. Because of the second mutation, R-2-HG can promote leukemogenesis [25-27], although the process is reversible [28]. IDH mutations were found to be stable during the evolution of AML with an incidence rate of 20\% [29-32]. In AML, heterozygous IDH mutations are noted including IDH1 R132H/C, IDH2 R140Q, and R172K [19]. The incidences of IDH1 and IDH2 mutations are equivalent and mutually exclusive [19, 33-36]; however, the incidence rate of IDH2 R140Q was found to be higher than that of IDH2 R172K (9.2\% vs. $2.9 \%$ ) [35]. IDH mutations are mostly found in the intermediate-risk karyotype group, especially in the normal karyotype, on the other hand, IDH mutations always accompanied with NPM1 mutation [16, 29, 36], but is mutually exclusive with the TET2 mutation [37]. Further,

(c) The Author(s). 2019 Open Access This article is distributed under the terms of the Creative Commons Attribution 4.0 International License (http://creativecommons.org/licenses/by/4.0/), which permits unrestricted use, distribution, and 
IDH2 R172 and NPM1 mutations were not detected in the same patient samples $[29,38]$. As shown in Table 1, different mutation isoforms have different prognostic values in AML, and remain controversial [29-31, 36, 39-47]. Some AML patients with IDH mutation, especially IDH2 R172 mutation, have a poor response to traditional chemotherapy and have a higher relapse rate [42]. Therefore, individualized treatment, especially targeted therapy for IDH mutations, may be an important option for such patients. In recent years, IDH inhibitors have shown good clinical response in AML patients. Based on phase 1/2 clinical trials, enasidenib and ivosidenib have been approved by the Food and Drug Administration (FDA) on 1 August 2017 and 20 July 2018 for the treatment of adult R/R AML with IDH2 and IDH1 mutations, respectively [48, 49]. This article provides a summary of the use of IDH inhibitors in AML with IDH mutations.

\section{IDH2 inhibitors \\ AGI-6780}

AGI-6780 is the first small selectively allosteric inhibitor of IDH2 R140Q mutation developed by Agios Pharmaceuticals in 2013. It could reverse the abnormal methylation of histones and DNA, and reduce the 2-HG level that is caused by the mutant IDH2 R140Q in TF-1 cell lines [50], as well as in primary AML cells, thus leading to the differentiation of AML cells [51]. Lack of in vivo test along with good clinical response with the subsequently developed drug enasidenib limited the clinical application of AGI-6780 although it could reduce the abnormal 2-HG level, reverse cell phenotypic changes, and induce differentiation of the primary AML cells in vitro.

\section{Enasidenib (AG-221, Idhifa $\left.{ }^{\circledR}\right)$}

Enasidenib (Idhifa ${ }^{\circ}$ ) is an oral selectively allosteric inhibitor of IDH2 mutation developed by Celgene Corporation under a global exclusive license from Agios Pharmaceuticals. It shows more powerful inhibitory effects on IDH2 R172K rather than IDH1 R140Q (ORR, 53.3\% vs. $35.4 \%$; CR, $24.4 \%$ vs. $17.7 \%)$ mutation, although it has inhibitory effects on both the mutations [52]. FDA has approved enasidenib for the treatment of adult $R / R$ AML with IDH2 mutations on 1 August 2017. The recommended oral dose is $100 \mathrm{mg}$ once daily for at least 6 months or until disease progression or intolerable adverse reaction [53].

Enasidenib could reduce the serum total 2-HG level by more than $90 \%$, reverse abnormal epigenetic changes, and induce differentiation of mutant IDH2 AML cells both in vitro and in murine xenograft models. Furthermore, it shows no cytotoxicity [54]. The results from a phase 1/2 clinical trial (NCT01915498) in adult R/R AML have shown that enasidenib monotherapy in adult $\mathrm{R} / \mathrm{R}$ AML is efficacious and safe. When patients were administered with oral enasidenib once daily for 28 days in a cycle, an overall response rate (ORR) was $40.3 \%$. About $87.3 \%$ of the patients reached their first treatment response within 5.8 months. The total $\mathrm{CR}$ rate was $19.3 \%$ with a median overall survival (OS) of 9.3 months and a median effect-free survival of 6.4 months, moreover, patients who reached CR had a longer OS of 19.7

Table 1 The treatment response and prognostic value of IDH mutations in adult AML patients

\begin{tabular}{|c|c|c|c|c|c|c|c|c|c|}
\hline Reference & & $\%($ Mut/All) & $\begin{array}{l}\text { Common } \\
\text { co-mutation }\end{array}$ & $C R+C R i(\%)$ & RR & OS & RFS & $\begin{array}{l}\text { Prognostic } \\
\text { value }\end{array}$ & Disease \\
\hline [29] & $\mathrm{IDH} 2$ & $12.1(54 / 446)$ & - & 72.2 & - & 12y-OS, median not reached & - & Favorable & AML \\
\hline [30] & IDH1 & $5.5(27 / 493)$ & - & 72.4 & - & - & - & No value & AML \\
\hline [39] & IDH1 R132 & $8(107 / 1333)$ & NPM1+ & 81 & 10y: 55\%; & 10y: $34 \%$ & 10y: 30\% & No value & $\mathrm{AML}$ \\
\hline [42] & IDH2 R140 & $8(119 / 1473)$ & NPM1+ & 88 & $5 y: 28 \%, 10 y: 35 \%$ & $5 y: 57 \%, 10 y: 46 \%$ & - & Favorable & AML \\
\hline [42] & IDH2 R172 & $2(29 / 1473)$ & None & 48 & 5y: 70\%, 10y: 70\% & $5 y: 24 \%, 10 y: 9 \%$ & - & Advers & AML \\
\hline [44] & IDH1 R132 & $16(34 / 213)$ & $\mathrm{NPM} 1+$ & 76 & $5 y: 64 \%$ & $4 y: 46 \%$ & - & No value & $\mathrm{CN}-\mathrm{AML}$ \\
\hline [44] & IDH2 R172 & $6(12 / 205)$ & None & 58 & $5 y: 100 \%$ & $4 y: 0 \%$ & - & Poor & CN-AML \\
\hline [45] & IDH1 R132 & $14(49 / 358)$ & $\mathrm{NPM} 1+$ & 73 & - & $3 y: 29 \%$ & - & No value & CN-AML \\
\hline [45] & IDH2 R140 & $16(56 / 358)$ & NPM1+ & 70 & - & $3 y: 39 \%$ & - & No value & CN-AML \\
\hline [45] & IDH2 R172 & $4(13 / 358)$ & None & 38 & - & $3 y: 0 \%$ & - & Poor & CN-AML \\
\hline [46] & IDH1 R132 & $7.5(32 / 426)$ & - & 91 & - & Median-OS: 20.9\% & - & poor & $\mathrm{AML}$ \\
\hline [46] & IDH2 R140 & $7.3(31 / 426)$ & - & 74 & - & Median-OS: 35.8\% & - & - & $\mathrm{AML}$ \\
\hline [46] & IDH2 R172 & $2.6(11 / 423)$ & - & 100 & - & Median-OS: 41.1\% & - & Favorable & AML \\
\hline [47] & IDH2 R172 & $1(18 / 1540)$ & None & - & - & $8 y$-OS: about $60 \%$ & - & Favorable & AML \\
\hline
\end{tabular}

$C R$ complete remission, $C R i C R$ with incomplete hematologic recovery, $O S$ overall survival, $R F S$ relapse-free survival, $R R$ relapse rate, $C N$ - $A M L$ cytogenetic normal AML 
months. Enasidenib seemed to induce AML cell differentiation and showed no cytotoxicity. Treatment-emergent adverse events were mainly elevated bilirubin (81\%), nausea (50\%), diarrhea (43\%). decreased appetite (34\%), and vomiting (34\%). The treatment-related grade $\geq 3$ adverse events occurred in $5 \%$ of the patients, and the most common were the elevation of indirect bilirubin level, nausea, differentiation syndrome (DS), tumor lysis syndrome, etc. However, with the continued use of the drug, the incidence of adverse reactions was gradually reduced. IDH-inhibitor-associated differentiation syndrome (IDH-DS) was reported in patients treated with enasidenib (IDH-DS of any grade occurred in $14 \%$ of patients, grade $\geq 3$ occurred in $7 \%$ of patients), and perhaps the myeloid differentiation was the reason. Alltrans retinoic acid (ATRA)-related DS occurred in acute promyelocytic leukemia (APL) with the incidence of 2$31 \%$, It was thought that inflammatory cytokines and rapidly infiltrated by mature cells lead to the DS, although the pathophysiology of ATRA-related DS is not entirely understood [55]. It remains unclear whether IDH-DS has the same mechanism with the DS occurred in APL, although they have the similar incidence, clinical manifestation, and both could be cured with glucocorticoids, hydroxyurea, and diuretics [56]. A phase 3 randomized, open-label study comparing the efficacy and safety of AG-221 vs. conventional care regimens (CCR) in older R/R AML patients ( $\geq$ 60 years) with an IDH2 mutations is presently ongoing (NCT02577406). The initial results have shown that the median OS in the enasidenib group was longer than that in the CCR group (8 months vs. 5 months) [57].

Enasidenib combined with standard chemotherapy or the hypomethylating agent (HMA) azacytidine is well tolerated and effective in AML patients with IDH2 mutation. The initial results of an ongoing clinical trial of enasidenib combined with the standard induction chemotherapy (NCT02632708) in the treatment of newly diagnosed AML patients with IDH2 mutations showed that the ORR was $62 \%$, the CR rate was $50 \%$, and the less 30 -days and 60 days mortality rate were 5 and $8 \%$, respectively $[58,59]$. Azacytidine and IDH inhibitors can directly or indirectly reduce DNA methylation levels and have synergistic effects on inducing cell differentiation. These characteristics have inspired an ongoing phase $1 / 2$ clinical trial of the combination of the two agents (NCT02677922), and the initial results indicated an ORR of $50 \%$ (3/6), one patient was withdrawn because of disease progression, while two others are still in a stable state [60]. A phase 2 clinical trial of enasidenib combined with azacytidine for the treatment of R/R AML with IDH2 mutations (NCT03683433) and a phase 1 clinical trial of enasidenib as maintenance therapy in post-HSCT AML patients with IDH2 mutations (NCT03728335) are ongoing. The ongoing clinical trials of enasidenib in adult AML are shown in Table 2.

\section{IDH1 inhibitors}

Different IDH1 inhibitors are effective against different types of IDH1 mutation. The ongoing clinical trials of IDH1 inhibitors in adult AML are shown in Table 3.

\section{Ivosidenib (AG-120, Tibsovo ${ }^{\circledR}$ )}

The unacceptable pharmacodynamic and pharmacokinetic properties have limited the clinical applications of AGI-5198, and AG-120 was the drug developed after due optimization [61]. Ivosidenib $\left(\right.$ Tibsovo $\left.^{\circ}\right)$ is a small molecule developed by Agios Pharmaceuticals and can be orally administered. It is an inhibitor of mutated IDH1 and could reduce the total serum 2-HG level and induce mutated IDH1 AML cell differentiation. It was approved by the FDA for the treatment of adult R/R AML with IDH1 mutations on 20 July 2018. The recommended dose is $500 \mathrm{mg}$ orally once daily (the dose can be adjusted according to the patient's condition)

Table 2 Ongoing Clinical trials of enasidenib in adult AML

\begin{tabular}{llll}
\hline NCT Number & Phase & disease & Interventions \\
\hline NCT03683433 & Phase 2 & R/R AML; & Enasidenib plus Azacitidine \\
NCT03881735 & Phase 2 & R/R AML; & Enasidenib; \\
NCT03825796 & Phase 2 & R/R AML & Enasidenib plus CPX-351 \\
NCT01915498 & Phase 1/2 & Advanced AML & enasidenib $\quad$ Recruiting \\
NCT03013998 & Phase 1/2 & Previously Untreated AML & enasidenib $\quad$ Active, not recruiting \\
NCT02632708 & Phase 1 & Newly Diagnosed AML & Enasidenib plus standard chemotherapy \\
NCT02677922 & Phase 1/2 & Newly Diagnosed AML & Enasidenib plus Azacitidine \\
NCT03839771 & Phase 3 & Newly Diagnosed AML; & AG-221 plus standard chemotherapy \\
NCT03515512 & Phase 1 & Post-HSCT AML & Ective,not recruiting \\
NCT03728335 & Phase 1 & Post-HSCT AML & Active,not recruiting \\
NCT02577406 & Phase 3 & AML $\geq 60$ years & enasidenib as maintenance therapy after HSCT
\end{tabular}


Table 3 Ongoing clinical trials of IDH1 inhibitors in adult AML

\begin{tabular}{|c|c|c|c|c|}
\hline NCT Number & Phase & disease & Interventions & Status \\
\hline NCT04013880 & Phase $1 / 2$ & R/R AML & FT-2102 plus ASTX727 & Not yet recruiting \\
\hline NCT02719574 & Phase $1 / 2$ & AML & $\begin{array}{l}\text { FT-2102 as single agent; } \\
\text { FT-2102 plus azacitidine/ cytarabine; }\end{array}$ & Recruiting \\
\hline NCT03471260 & Phase 1/2 & $\mathrm{R} / \mathrm{R}$ AML & $\begin{array}{l}\text { Ivosidenib plus Venetoclax with or } \\
\text { without azacitidine }\end{array}$ & Recruiting \\
\hline NCT02074839 & Phase 1 & $\begin{array}{l}\text { R/R AML; } \\
\text { Untreated AML; }\end{array}$ & ivosidenib & Active, not recruiting \\
\hline NCT02677922 & Phase $1 / 2$ & Newly Diagnosed AML & Ivosidenib plus Azacitidine & Active, not recruiting \\
\hline NCT03173248 & Phase 3 & Untreated AML & $\begin{array}{l}\text { Ivosidenib plus azacitidine vs placebo } \\
\text { plus azacitidine }\end{array}$ & Recruiting \\
\hline NCT02632708 & Phase 1 & Newly Diagnosed AML & Ivosidenib plus standard chemotherapy & Active, not recruiting \\
\hline NCT03839771 & Phase 3 & Newly Diagnosed AML & Ivosidenib plus standard chemotherapy & Recruiting \\
\hline NCT03013998 & Phase $1 / 2$ & Previously Untreated AML & ivosidenib & Recruiting \\
\hline
\end{tabular}

ASTX727 is an oral deoxyribonucleic acid (DNA) methyltransferase (DNMT) inhibitor

until disease progression or occurrence of unacceptable toxicity or HSCT [49].

The approval of ivosidenib by the FDA was based on a phase $1 / 2$ clinical trial of ivosidenib monotherapy in adult R/R AML with IDH1 mutations (NCT02074839). The trial concluded that ivosidenib monotherapy was safe and effective and it could reduce or even eliminate variant allele frequency (VAF) so that some patients could achieve a deep and lasting remission. In this clinical trial, the ORR was $41.6 \%$ with a median response time of 6.5 months, and the CR rate was $21.6 \%$ with a median duration time of 9.3 months. The patients who reached molecular remission had a longer CR duration than those who did not. The CR + CRi (CR with incomplete hematologic recovery) rate was $30.4 \%$, thus leading to $35 \%$ of the patients being independent of blood transfusion. Ivosidenib induce AML cell differentiation and seems no cytotoxicity. Adverse events occurred in 99\% patients treated with ivosidenib. Mainly included diarrhea (31\%), leukocytosis (30\%), febrile neutropenia (29\%), nausea (28\%), fatigue (26\%), dyspnea (25\%), prolonged QT interval (25\%), peripheral edema (22\%), anemia (22\%), fever $(21 \%)$ and cough (21\%). There were no adverse events related deaths, although the treatment-related grade $\geq 3$ adverse events occurred in $21 \%$ of patients, and the most common were prolonged QT interval, DS and leukocytosis, which were also the most common reasons for drug withdrawal. Treatment with hydroxyurea or leukapheresis could be initiated for leukocytosis. IDH-DS occurred in about $11 \%$ of the patients with a median onset time of 29 days, and could be cured by temporary withdrawal of the drug and adding glucocorticoids and diuretics [34, 62, 63].

The ongoing clinical trial of ivosidenib combined with standard therapy or azacytidine for the treatment of adult R/R AML showed promising results. Ivosidenib combined with standard therapy was safe and effective in both primary and secondary AML (NCT02632708), although the ORR was higher in the former $(86 \%$ vs. $44 \%$ ) [59]. The CR rate was $3 / 5$ (the other two patients had a stable disease) in a clinical trial of ivosidenib combined with azacytidine for the treatment of AML (NCT02677922) [60].

A global phase 3 clinical trial evaluating the efficacy and safety of ivosidenib combined with azacytidine vs. placebo combined with azacytidine in newly diagnosed AML patients with IDH1 mutations who are not suitable for intense chemotherapy is ongoing (NCT03173248). A phase $1 / 2$ clinical trial of ivosidenib combined with venetoclax with or without azacytidine is also ongoing (NCT03471260).

\section{BAY1436032}

BAY1436032 is an orally administered allosteric inhibitor of all the IDH1 mutation proteins. It can pass through the blood-brain barrier [64]. BAY1436032 monotherapy can reduce the $2-\mathrm{HG}$ level to a maximum, inhibit the self-renewal and proliferation of leukemia stem cells, and reverse the abnormal methylation of histone in patient-derived xenograft (PDX) mouse models [65]. BAY1436032 combined with azacytidine induced AML cell differentiation by regulating the EGR-GFI1NFKB pathway [66]. A single arm non-randomized, multicenter phase $1 / 2$ clinical trial of BAY1436032 for the treatment of advanced AML has been completed, but the results have not been reported yet (NCT03127735).

\section{FT-2102}

FT-2102 is an inhibitor of the mutated IDH1. A phase 1/2 clinical trial of FT-2102 monotherapy and combined therapy with azacytidine or cytarabine for the treatment of adult R/R AML is ongoing (NCT02719574). The phase 1 results indicated the efficacy and safety of the 
drug. The ORR was $33 \%$ and the CR rate was $14 \%$, and there were no deaths related to FT-2102. The main adverse reactions were thrombocytopenia, febrile neutropenia, anemia, pneumonia, etc. There were no deaths related to FT-2102. Like for other IDH inhibitors, IDH-DS was reported, but it could be cured by withdrawing the drug and adding dexamethasone and hydroxyurea. Based on the phase 1 data, FT-2102 at a dose of $150 \mathrm{mg}$ twice daily has been administered in the ongoing phase 2 clinical trial [67]. In addition, a phase $1 / 2$ clinical trial of FT-2102 combined with DNA methyltransferase (DNMT) inhibitor for the treatment of R/R AML with IDH R132 mutation is presently ongoing (NCT04013880).

\section{IDH305}

IDH305 is a selective allosteric inhibitor of IDH1 mutation. It is an optimized product of IDH889. Preclinical studies have indicated that it could reduce the 2-HG level and induce differentiation of AML cells [68]. A phase 1 clinical trial of IDH305 for the treatment of advanced malignancies with IDH R132 is ongoing (NCT02381886). The initial results have revealed a good clinical effect. The ORR, CR rate, Cri, and PR rate were 33\% (7/21), 9.5\% (2/ $21), 4.8 \%(1 / 21)$, and $19 \%$, respectively. The adverse events were mainly raised bilirubin and lipase [69]. Further studies evaluating the safety and tolerability of IDH305 as a single agent and in combination were needed.

\section{Other IDH1 inhibitors}

The other IDH1 inhibitors include AGI-5198, ML309 (AGI-5027), GSK 321, and DC_H31. AGI-5198 is a selective IDH1 R132H inhibitor [70], The pharmacokinetic properties of this drug (rapid metabolism and clearance) have limited its research and application value [64]. ML309 and GSK 321 have been mainly tried in solid tumors at present, although both are selective inhibitors of the mutant IDH1 [35, 71]. Recently, a new small molecular allosteric inhibitor of IDH1 R132H/C, called DC_H31, has been developed. It could reduce the 2-HG level and inhibit cell proliferation and promote cell differentiation in HT1080 cell lines [72], However, in vivo trials are needed to validate these effects to develop this drug as a promising new targeted inhibitor shortly.

\section{Pan-IDH inhibitor AG-881}

Developed by Agios Pharmaceuticals, AG-881 is a panIDH inhibitor that shows inhibitory effects to both IDH1 and IDH2 mutations. The mutated IDH1 and IDH2 bind to AG-881 at different residues in a similar way due to different allosteric inhibitory regions. AGI-881 combined with IDH1 R132H and IDH2 R172K acts faster than IDH2 R140. The drug could easily pass through the blood-brain barrier and induce myeloid differentiation, thereby making it a more promising IDH inhibitor [73, 74]. A phase 1 clinical trial evaluating the efficacy and safety of AGI-881 in myeloid malignancies with mutated IDH1/IDH2 is presently ongoing (NCT02492737).

\section{Indirect IDH inhibitors \\ Venetoclax}

Abnormal accumulation of R-2-HG inhibits cytochrome c oxidase (COX) producing an environment similar to hypoxia, and thus, activates the apoptotic precursor proteins BAX and BAK through the BCL-2 homologous effector. The mitochondrial outer membrane becomes transparent and ultimately apoptosis is promoted. However, the antiapoptotic gene BCL-2 could antagonize BAX and BAK, thereby preventing the increased permeability of the mitochondrial outer membrane [75]. This promotes cell survival, and the mutated IDH cells show dependence on BCL-2 [76, 77]. Venetoclax is a highly selective BCL-2 inhibitor. Venetoclax monotherapy in IDH1/IDH2 mutated AML patients led to a CR + CRi up to 33\% [78]. However, when combined with decitabine or azacytidine, the CR + CRi were 33 and 59\% in mutated IDH R/R AML patients and AML patients not suitable for intensive treatment, respectively $[76,79]$. When venetoclax was combined with decitabine or azacytidine for the treatment of older AML patients, the CR + CRi was $71 \%$ with a median OS of 24.4 months [80]. Therefore, venetoclax is a promising new drug for the treatment of AML.

\section{Inhibitors of glutaminase and glutamate dehydrogenase (GLUD)}

${ }^{13} \mathrm{C}$-tracing studies have shown that the carbon atoms of 2-HG in AML cells with IDH mutations are mainly derived from glutamine or glutamate. Glutamine is converted to glutamate catalyzed by glutaminase, and then GLUD catalyzes the oxidative decarboxylation of glutamate to produce $\alpha-K G$. Finally, $\alpha-K G$ is converted to $2-\mathrm{HG}$ in the mutated IDH cells [81, 82]. The inhibitors of glutaminase could jeopardize the metabolism of glutamine as a consequence of selective inhibition of the proliferation of the IDH mutant AML cells in vitro [83]. In the HCT116 colorectal cancer cells, the use of glutamate dehydrogenase inhibitor epigallocatechin-3gallate EGCG could reduce the 2-HG level and inhibit the proliferation of the IDH mutant cells [82]; however, this should be further verified in AML cell lines.

\section{Target of HIF-1a}

In IDH mutant cells, the abnormal accumulation of R-2HG could down-regulate the levels of HIF-1 $\alpha$ by activating proline 4-hydroxylase EgIN as a result of inhibition of cell differentiation. This may be one of the factors driving leukemogenesis [28, 84-86]. Thus, in the IDH mutant cancer cells, the target of HIF- $1 \alpha$ may be a new treatment choice for AML therapy [27]. 


\section{Resistance to the IDH inhibitors}

There were some problems in the clinical applications of the IDH inhibitors in AML patients with mutations, such as primary and acquired resistance, although IDH inhibitors could provide in these patients an average survival time of 18-21 months [87]. Among patients who had primary resistance, there were some mutations in the NRAS and MAPK pathways, which might be the causes of primary resistance $[38,54]$.

The mechanisms of acquired resistance remain unclear. One study found that in R/R AML patients with IDH2 R140Q mutation treated with enasidenib, there are second site IDH2 mutations in the trans-state [Q316E mutation (at glutamine 316 position) and I319M mutation (at isoleucine 319 position) when they had acquired resistance [88]. Another study found that two R/R AML patients with IDH1 R132C mutation had achieved CR after treatment with ivosidenib. The final relapse was because of the acquired resistance to ivosidenib, and the IDH2 R140Q mutation was detected with the relapse [89]. Likewise, IDH1 R132C or R132H mutations were detected in the mutant IDH2 AML patients who had acquired resistance to enasidenib [87]. In summary, we speculate that the second site mutations of the allele and the mutual conversion of IDH mutant subtypes are the two mechanisms leading to the acquired resistance to the IDH inhibitors.

\section{Conclusions}

Enasidenib and ivosidenib have been approved by the FDA for the treatment of adult R/R AML with mutant IDH2 and mutant IDH1, respectively, due to their high treatment response and absence of cytotoxicity in clinical trials $[48,49]$. However, there exist some problems, such as resistance. Hence, a combined therapy and the pan-IDH inhibitors may, to some degree, resolve the question of acquired resistance to IDH inhibitors [89]. Meanwhile, racial and ethnic disparities of IDH mutations should also be considered and studied [90]. IDH mutations are often accompanied by abnormal methylation of DNA, and hence, IDH inhibitors combined with demethylating agents may become one of the main therapeutic ways for the treatment of IDH mutant AML patients. Because IDH mutations are the early mutations in AML, therefore, the application of IDH inhibitors should not only be limited to R/R AML patients but also all AML patients having IDH mutations. Clinical trials of the IDH inhibitors combined with standard chemotherapy or HMA for the treatment of R/R AML or newly diagnosed AML are ongoing. Furthermore, clinical trials evaluating the clinical efficacy of IDH inhibitors as maintenance therapy in postHSCT AML patients are also ongoing.

\begin{abstract}
Abbreviations
AML: Acute myeloid leukemia; APL: Acute promyelocytic leukemia; ATRA: Alltrans retinoic acid; CN-AML: Cytogenetic normal AML; COX: Cytochrome c oxidase; CR: Complete remission; CRi: CR with incomplete hematologic recovery; DS: Differentiation syndrome; FDA: Food and Drug Administration; HMA: Hypomethylating agents; HSCT: Hematopoietic stem cell transplantation; IDH: Isocitrate dehydrogenase; ORR: Overall response rate; OS: Overall survival; PDX: Patient derived Xenograft; R/R AML: Relapsed or refractory AML; RFS: Relapse-free survival; RR: Relapse rate; TCA: Tricarboxylic acid cycle; VAF: Variant allele frequency; a-KG: a-ketoglutarate
\end{abstract}

\section{Acknowledgements \\ Not applicable.}

\section{Authors' contributions}

Both authors have contributed to write and revise the manuscripts. Both authors have read and approved the final version.

\section{Funding}

This work was supported by the Foundation of the Science \& Technology Department of Sichuan Province (NO.2019YFS0026).

\section{Availability of data and materials}

Not applicable.

Ethics approval and consent to participate

Not applicable.

\section{Consent for publication}

Not applicable.

\section{Competing interests}

The authors declare that they have no competing interests.

Received: 9 August 2019 Accepted: 30 September 2019

Published online: 22 October 2019

\section{References}

1. Bose P, Vachhani P, Cortes JE. Treatment of relapsed/refractory acute myeloid leukemia. Curr Treat Options in Oncol. 2017;18(3):17.

2. Dohner H, Weisdorf DJ, Bloomfield CD. Acute Myeloid Leukemia. N Engl J Med. 2015;373(12):1136-52.

3. Roboz GJ, Rosenblat T, Arellano M, Gobbi M, Altman JK, Montesinos P, et al. International randomized phase III study of elacytarabine versus investigator choice in patients with relapsed/refractory acute myeloid leukemia. J Clin Oncol. 2014;32(18):1919-26.

4. Walter RB, Estey EH. Management of older or unfit patients with acute myeloid leukemia. Leukemia. 2015;29(4):770-5

5. Gu R, Yang X, Wei H. Molecular landscape and targeted therapy of acute myeloid leukemia. Biomark Res. 2018;6:32.

6. Zhang W, Xu J. DNA methyltransferases and their roles in tumorigenesis. Biomark Res. 2017:5:1.

7. Yang $X$, Wang J. Precision therapy for acute myeloid leukemia. J Hematol Oncol. 2018;11(1):3.

8. Saygin C, Carraway HE. Emerging therapies for acute myeloid leukemia. J Hematol Oncol. 2017;10(1):93.

9. Wu M, Li C, Zhu X. FLT3 inhibitors in acute myeloid leukemia. J Hematol Oncol. 2018;11(1):133.

10. Wang Y, Xu Y, Li S, Liu J, Xing Y, Xing H, et al. Targeting FLT3 in acute myeloid leukemia using ligand-based chimeric antigen receptor-engineered T cells. J Hematol Oncol. 2018;11(1):60.

11. Ling $Y$, Xie $Q$, Zhang Z, Zhang H. Protein kinase inhibitors for acute leukemia. Biomark Res. 2018:6:8.

12. Zhou J, Toh SH, Chan ZL, Quah JY, Chooi JY, Tan TZ, et al. A loss-of-function genetic screening reveals synergistic targeting of AKT/mTOR and WTN/betacatenin pathways for treatment of AML with high PRL-3 phosphatase. J Hematol Oncol. 2018;11(1):36.

13. Reitman ZJ, Yan H. Isocitrate dehydrogenase 1 and 2 mutations in cancer: alterations at a crossroads of cellular metabolism. J Natl Cancer Inst. 2010; 102(13):932-41. 
14. Parsons DW, Jones S, Zhang X, Lin JC, Leary RJ, Angenendt P, et al. An integrated genomic analysis of human glioblastoma multiforme. Science. 2008;321(5897):1807-12.

15. Yan H, Parsons DW, Jin G, McLendon R, Rasheed BA, Yuan W, et al. IDH1 and IDH2 mutations in gliomas. N Engl J Med. 2009;360(8):765-73.

16. Mardis ER, Ding L, Dooling DJ, Larson DE, McLellan MD, Chen K, et al. Recurring mutations found by sequencing an acute myeloid leukemia genome. N Engl J Med. 2009;361(11):1058-66.

17. Kipp BR, Voss JS, Kerr SE, Barr Fritcher EG, Graham RP, Zhang L, et al. Isocitrate dehydrogenase 1 and 2 mutations in cholangiocarcinoma. Hum Pathol. 2012;43(10):1552-8.

18. Amary MF, Bacsi K, Maggiani F, Damato S, Halai D, Berisha F, et al. IDH1 and $\mathrm{IDH} 2$ mutations are frequent events in central chondrosarcoma and central and periosteal chondromas but not in other mesenchymal tumours. J Pathol. 2011:224(3):334-43.

19. Ward PS, Patel J, Wise DR, Abdel-Wahab O, Bennett BD, Coller HA, et al. The common feature of leukemia-associated IDH1 and IDH2 mutations is a neomorphic enzyme activity converting alpha-ketoglutarate to 2hydroxyglutarate. Cancer Cell. 2010;17(3):225-34.

20. Rakheja D, Medeiros $L J$, Bevan S, Chen W. The emerging role of $d-2$ hydroxyglutarate as an oncometabolite in hematolymphoid and central nervous system neoplasms. Front Oncol. 2013;3:169.

21. Chowdhury R, Yeoh KK, Tian YM, Hillringhaus L, Bagg EA, Rose NR, et al. The oncometabolite 2-hydroxyglutarate inhibits histone lysine demethylases. EMBO Rep. 2011;12(5):463-9.

22. Xu W, Yang H, Liu Y, Yang Y, Wang P, Kim SH, et al. Oncometabolite 2hydroxyglutarate is a competitive inhibitor of alpha-ketoglutaratedependent dioxygenases. Cancer Cell. 2011;19(1):17-30.

23. Lu C, Ward PS, Kapoor GS, Rohle D, Turcan S, Abdel-Wahab O, et al. IDH mutation impairs histone demethylation and results in a block to cell differentiation. Nature. 2012;483(7390):474-8.

24. Figueroa ME, Abdel-Wahab O, Lu C, Ward PS, Patel J, Shih A, et al. Leukemic $\mathrm{IDH} 1$ and IDH2 mutations result in a hypermethylation phenotype, disrupt TET2 function, and impair hematopoietic differentiation. Cancer Cell. 2010; 18(6):553-67.

25. Chaturvedi A, Araujo Cruz MM, Jyotsana N, Sharma A, Yun H, Gorlich K, et al. Mutant IDH1 promotes leukemogenesis in vivo and can be specifically targeted in human AML. Blood. 2013;122(16):2877-87.

26. Kats LM, Reschke M, Taulli R, Pozdnyakova O, Burgess K, Bhargava P, et al. Proto-oncogenic role of mutant IDH2 in leukemia initiation and maintenance. Cell Stem Cell. 2014;14(3):329-41.

27. Molenaar RJ, Radivoyevitch T, Maciejewski JP, van Noorden CJ, Bleeker FE. The driver and passenger effects of isocitrate dehydrogenase 1 and 2 mutations in oncogenesis and survival prolongation. Biochim Biophys Acta. 2014;1846(2):326-41. https://doi.org/10.1016/j.bbcan.2014. 05.004.

28. Losman JA, Looper RE, Koivunen P, Lee S, Schneider RK, McMahon C, et al. (R)-2-hydroxyglutarate is sufficient to promote leukemogenesis and its effects are reversible. Science. 2013;339(6127):1621-5.

29. Chou WC, Lei WC, Ko BS, Hou HA, Chen CY, Tang JL, et al. The prognostic impact and stability of Isocitrate dehydrogenase 2 mutation in adult patients with acute myeloid leukemia. Leukemia. 2011;25(2): 246-53.

30. Chou WC, Hou HA, Chen CY, Tang JL, Yao M, Tsay W, et al. Distinct clinical and biologic characteristics in adult acute myeloid leukemia bearing the isocitrate dehydrogenase 1 mutation. Blood. 2010;115(14):2749-54.

31. Thol F, Damm F, Wagner K, Gohring G, Schlegelberger B, Hoelzer D, et al. Prognostic impact of IDH2 mutations in cytogenetically normal acute myeloid leukemia. Blood. 2010;116(4):614-6.

32. Fathi AT, Sadrzadeh H, Borger DR, Ballen KK, Amrein PC, Attar EC, et al. Prospective serial evaluation of 2-hydroxyglutarate, during treatment of newly diagnosed acute myeloid leukemia, to assess disease activity and therapeutic response. Blood. 2012;120(23):4649-52.

33. Benton CB, Ravandi F, Andreeff M, Kadia T, Ruvolo V, Qiu P, et al. Case series of patients with acute myeloid leukemia receiving hypomethylation therapy and retrospectively found to have IDH1 or IDH2 mutations. Leuk Lymphoma. 2014;55(6):1431-4.

34. Norsworthy KJ, Luo L, Hsu V, Gudi R, Dorff SE, Przepiorka D, et al. FDA approval summary: Ivosidenib for relapsed or refractory acute myeloid leukemia with an Isocitrate Dehydrogenase-1 mutation. Clin Cancer Res. 2019;25(11):3205-9.
35. Sharma H. Development of novel therapeutics targeting Isocitrate dehydrogenase mutations in Cancer. Curr Top Med Chem. 2018;18(6): 505-24.

36. Abbas S, Lugthart S, Kavelaars FG, Schelen A, Koenders JE, Zeilemaker A et al. Acquired mutations in the genes encoding IDH1 and IDH2 both are recurrent aberrations in acute myeloid leukemia: prevalence and prognostic value. Blood. 2010;116(12):2122-6.

37. Gaidzik VI, Paschka P, Spath D, Habdank M, Kohne CH, Germing U, et al. TET2 mutations in acute myeloid leukemia (AML): results from a comprehensive genetic and clinical analysis of the AML study group. J Clin Oncol. 2012:30(12):1350-7.

38. Stein EM, DiNardo D, Fathi AT, Pollyea DA, Stone RM, Altman JK, et al. Molecular remission and response patterns in patients with mutant-IDH2 acute myeloid leukemia treated with enasidenib. Blood. 2019;133(7):676-87. https://doi.org/10.1182/blood-2018-08-869008.

39. Green CL, Evans CM, Hills RK, Burnett AK, Linch DC, Gale RE. The prognostic significance of IDH1 mutations in younger adult patients with acute myeloid leukemia is dependent on FLT3/ITD status. Blood. 2010;116(15): 2779-82.

40. Wagner K, Damm F, Gohring G, Gorlich K, Heuser M, Schafer I, et al. Impact of IDH1 R132 mutations and an IDH1 single nucleotide polymorphism in cytogenetically normal acute myeloid leukemia: SNP rs11554137 is an adverse prognostic factor. J Clin Oncol. 2010;28(14):2356-64.

41. Xu Q, Li Y, LV N, Jing Y, XU Y, Li Y, et al. Correlation between Isocitrate dehydrogenase gene aberrations and prognosis of patients with acute myeloid leukemia: a systematic review and meta-analysis. Clin Cancer Res. 2017;23(15):4511-22.

42. Green $\mathrm{CL}$, Evans CM, Zhao L, Hills RK, Burnett AK, Linch DC, et al. The prognostic significance of $\mathrm{IDH} 2$ mutations in $\mathrm{AML}$ depends on the location of the mutation. Blood. 2011:118(2):409-12.

43. Largeaud L, Berard E, Bertoli S, Dufrechou S, Prade N, Gadaud N, et al. Outcome of AML patients with IDH2 mutations in real world before the era of IDH2 inhibitors. Leuk Res. 2019;81:82-7.

44. Boissel N, Nibourel O, Renneville A, Gardin C, Reman O, Contentin N, et al. Prognostic impact of isocitrate dehydrogenase enzyme isoforms 1 and 2 mutations in acute myeloid leukemia: a study by the acute leukemia French association group. J Clin Oncol. 2010;28(23):3717-23.

45. Marcucci G, Maharry K, Wu YZ, Radmacher MD, Mrozek K, Margeson D, et al. $\mathrm{IDH} 1$ and IDH2 gene mutations identify novel molecular subsets within de novo cytogenetically normal acute myeloid leukemia: a Cancer and leukemia group B study. J Clin Oncol. 2010;28(14):2348-55.

46. Bertoli S, Tavitian S, Delabesse E, Sarry A, Huguet F, Recher C. Outcome of AML patients with IDH1 or IDH2 mutations from diagnosis and refractory/ relapse phase of the disease in routine practice. Blood. 2016;128(22):1718.

47. Papaemmanuil E, Gerstung M, Bullinger L, Gaidzik VI, Paschka P, Roberts ND, et al. Genomic classification and prognosis in acute myeloid leukemia. N Engl J Med. 2016:374(23):2209-21.

48. Kim ES. Enasidenib: First Global Approval. Drugs. 2017;77(15):1705-11.

49. Dhillon S. Ivosidenib: First Global Approval. Drugs. 2018;78(14):1509-16.

50. Kernytsky A, Wang F, Hansen E, Schalm S, Straley K, Gliser C, et al. IDH2 mutation-induced histone and DNA hypermethylation is progressively reversed by small-molecule inhibition. Blood. 2015;125(2):296-303.

51. Wang F, Travins J, DeLaBarre B, Penard-Lacronique V, Schalm S, Hansen E, et al. Targeted inhibition of mutant IDH2 in leukemia cells induces cellular differentiation. Science. 2013;340:622-6.

52. Galkin M, Jonas BA. Enasidenib in the treatment of relapsed/refractory acute myeloid leukemia: an evidence-based review of its place in therapy. Core Evid. 2019;14:3-17.

53. Yen K, Travins J, Wang F, David MD, Artin E, Straley K, et al. AG-221, a firstin-class therapy targeting acute myeloid leukemia harboring oncogenic IDH2 mutations. Cancer Discov. 2017;7(5):478-93.

54. Amatangelo MD, Quek L, Shih A, Stein EM, Roshal M, David MD, et al. Enasidenib induces acute myeloid leukemia cell differentiation to promote clinical response. Blood. 2017:130(6):732-41.

55. Rogers JE, Yang D. Differentiation syndrome in patients with acute promyelocytic leukemia. J Oncol Pharm Pract. 2012;18(1):109-14.

56. Stein EM, DiNardo CD, Pollyea DA, Fathi AT, Roboz GJ, Altman JK, et al. Enasidenib in mutant $\mathrm{IDH} 2$ relapsed or refractory acute myeloid leukemia. Blood. 2017;130(6):722-31.

57. Tallman MS, Knight RD, Glasmacher AG, Dohner $\mathrm{H}$. Phase III randomized, open-label study comparing the efficacy and safety of AG-221 vs 
conventional care regimens (CCR) in older patients with advanced acute myeloid leukemia (AML) with isocitrate dehydrogenase (IDH)-2 mutations in relapse or refractory to multiple prior treatments: the IDHENTIFY trial. J Clin Oncol. 2016;34.

58. Amaya ML, Pollyea DA. Targeting the IDH2 pathway in acute myeloid leukemia. Clin Cancer Res. 2018;24(20):4931-6. https://doi.org/10.1158/10780432.CCR-18-0536.

59. Stein EM, DiNardo CD, Mims AS, Savona MR, Pratz K, Stein AS, et al. Ivosidenib or Enasidenib combined with standard induction chemotherapy is well tolerated and active in patients with newly diagnosed $\mathrm{AML}$ with an $\mathrm{IDH} 1$ or IDH2 mutation: initial results from a phase 1 trial. Blood. 2017;130:726.

60. DiNardo CD, Stein AS, Fathi AT, Montesinos P, Odenike O, Kantarjian HM, et al. Mutant Isocitrate dehydrogenase $(\mathrm{mIDH})$ inhibitors, Enasidenib or Ivosidenib, in combination with Azacitidine (AZA): preliminary results of a phase $1 \mathrm{~b} / 2$ study in patients with newly diagnosed acute myeloid leukemia (AML). Blood. 2017;130:639

61. Popovici-Muller J, Lemieux RM, Artin E, Saunders JO, Salituro FG, Travins J, et al. Discovery of AG-120 (Ivosidenib): a first-in-class mutant IDH1 inhibitor for the treatment of IDH1 mutant cancers. ACS Med Chem Lett. 2018;9(4):300-5.

62. DiNardo CD, Stein EM, de Botton S, Roboz GJ, Altman JK, Mims AS, et al. Durable remissions with Ivosidenib in IDH1-mutated relapsed or refractory AML. N Engl J Med. 2018;378(25):2386-98.

63. DiNardo CD, de Botton S, Stein EM, Roboz GJ, Swords RT, Pollyea DA, et al Determination of IDH1 mutational burden and clearance via nextgeneration sequencing in patients with $\mathrm{IDH} 1$ mutation-positive hematologic malignancies receiving AG-120, a first-in-class inhibitor of mutant IDH1. Blood. 2016:128:1070.

64. Golub D, lyengar N, Dogra S, Wong T, Bready D, Tang K, et al. Mutant Isocitrate dehydrogenase inhibitors as targeted Cancer therapeutics. Front Oncol. 2019;9:417.

65. Chaturvedi A, Herbst L, Pusch S, Klett L, Goparaju R, Stichel D, et al. Panmutant-IDH1 inhibitor BAY1436032 is highly effective against human IDH1 mutant acute myeloid leukemia in vivo. Leukemia. 2017;31(10):2020-8.

66. Chaturvedi A, Gupta C, Goparaju R, Gabdoulline R, Kaulfuss S, Görlich K, et al. Synergistic activity of IDH1 Inhibitor Bay-1436032 with Azacitidine in IDH1 mutant acute myeloid leukemia. Blood. 2017;130(Suppl 1):1352.

67. Watts J, Baer MR, Yang J, Dinner S, Lee S, Seiter K, et al. Phase 1 study of the IDH1m inhibitor FT-2102 as a single agent in patients with IDH1m acute myeloid leukemia (AML) or myelodysplastic syndrome (MDS). Blood. 2018; 132(Suppl 1):1453.

68. Cho YS, Levell JR, Liu G, Caferro T, Sutton J, Shafer CM, et al. Discovery and evaluation of clinical candidate IDH305, a brain penetrant mutant IDH1 inhibitor. ACS Med Chem Lett. 2017;8(10):1116-21.

69. DiNardo CD, Schimmer AD, Yee KW, Hochhaus A, Kraemer A, Carvajal RD, et al. A phase I study of IDH305 in patients with advanced malignancies including relapsed/refractory AML and MDS that harbor IDH1R132 mutations. Blood. 2016;128(22):1073 http://www.bloodjournal.org/ content/128/22/1073.

70. Rohle D, Popovici-Muller J, Palaskas N, Turcan S, Grommes C, Campos C, et al. An inhibitor of mutant IDH1 delays growth and promotes differentiation of glioma cells. Science. 2013;340(6132):626-30.

71. Davis M, Pragani R, Popovici-Muller J, et al. ML309: A potent inhibitor of $\mathrm{R} 132 \mathrm{H}$ mutant IDH1 capable of reducing 2-hydroxyglutarate production in U87 MG glioblastoma cells. 2012 Apr 16 [Updated 2013 May 8]. In: Probe Reports from the NIH Molecular Libraries Program. Bethesda: National Center for Biotechnology Information (US); 2010. Available from: https:// www.ncbi.n/m.nih.gov/books/NBK153220/

72. Duan Z, Liu J, Niu L, Wang J, Feng M, Chen H, et al. Discovery of DC_H31 as potential mutant IDH1 inhibitor through NADPH-based high throughput screening. Bioorg Med Chem. 2019;27(15):3229-36.

73. Ma R, Yun CH. Crystal structures of pan-IDH inhibitor AG-881 in complex with mutant human IDH1 and IDH2. Biochem Biophys Res Commun. 2018; 503(4):2912-7.

74. Yen K, Konteatis Z, Sui Z, Artin E, Dang L, Straley K, et al. Abstract B126: AG881, a brain penetrant, potent, pan-mutant IDH $(\mathrm{mIDH})$ inhibitor for use in mIDH solid and hematologic malignancies. Mol Cancer Ther. 2018;17(1 Supplement):B126

75. Perini GF, Ribeiro GN, Pinto Neto JV, Campos LT, Hamerschlak N. BCL-2 as therapeutic target for hematological malignancies. J Hematol Oncol. 2018; 11(1):65 .
76. Buege MJ, DiPippo AJ, DiNardo CD. Evolving Treatment Strategies for Elderly Leukemia Patients with IDH Mutations. Cancers (Basel). 2018;10(6): 187. https://doi.org/10.3390/cancers10060187.

77. Chan SM, Thomas D, Corces-Zimmerman MR, Xavy S, Rastogi S, Hong WJ, et al. Isocitrate dehydrogenase 1 and 2 mutations induce BCL-2 dependence in acute myeloid leukemia. Nat Med. 2015;21(2):178-84.

78. Konopleva M, Pollyea DA, Potluri J, Chyla B, Hogdal L, Busman T, et al. Efficacy and biological correlates of response in a phase II study of Venetoclax monotherapy in patients with acute myelogenous leukemia. Cancer Discov. 2016;6(10):1106-17.

79. Wei AH, Tiong IS. Midostaurin, enasidenib, CPX-351, gemtuzumab ozogamicin, and venetoclax bring new hope to AML. Blood. 2017;130(23): 2469-74.

80. DiNardo CD, Pratz K, Pullarkat V, Jonas BA, Arellano M, Becker PS, et al. Venetoclax combined with decitabine or azacitidine in treatment-naive, elderly patients with acute myeloid leukemia. Blood. 2019;133(1):7-17.

81. Dang L, White DW, Gross S, Bennett BD, Bittinger MA, Driggers EM, et al Cancer-associated IDH1 mutations produce 2-hydroxyglutarate. Nature. 2009;462(7274):739-44

82. Peeters TH, Lenting K, Breukels V, van Lith SAM, van den Heuvel CNAM, Molenaar $\mathrm{R}$, et al. Isocitrate dehydrogenase 1-mutated cancers are sensitive to the green tea polyphenol epigallocatechin-3-gallate. Cancer Metab. 2019;7(1). https://doi.org/10.1186/s40170-019-0198-7.

83. Emadi A, Jun SA, Tsukamoto T, Fathi AT, Minden MD, Dang CV. Inhibition of glutaminase selectively suppresses the growth of primary acute myeloid leukemia cells with IDH mutations. Exp Hematol. 2014;42(4):247-51.

84. Song LP, Zhang J, Wu SF, Huang Y, Zhao Q, Cao JP, et al. Hypoxia-inducible factor-1alpha-induced differentiation of myeloid leukemic cells is its transcriptional activity independent. Oncogene. 2008;27(4):519-27.

85. Liu W, Guo M, Xu YB, Li D, Zhou ZN, Wu YL, et al. Induction of tumor arrest and differentiation with prolonged survival by intermittent hypoxia in a mouse model of acute myeloid leukemia. Blood. 2006;107(2):698-707.

86. Koivunen P, Lee S, Duncan CG, Lopez G, Lu G, Ramkissoon S, et al. Transformation by the (R)-enantiomer of 2-hydroxyglutarate linked to EGLN activation. Nature. 2012;483(7390):484-8.

87. Quek L, David MD, Kennedy A, Metzner M, Amatangelo M, Shih A, et al. Clonal heterogeneity of acute myeloid leukemia treated with the IDH2 inhibitor enasidenib. Nat Med. 2018;24(8):1167-77.

88. Intlekofer AM, Shih AH, Wang B, Nazir A, Rustenburg AS, Albanese SK, et al. Acquired resistance to IDH inhibition through trans or cis dimer-interface mutations. Nature. 2018;559(7712):125-9.

89. Harding JJ, Lowery MA, Shih AH, Schvartzman JM, Hou S, Famulare C, et al. Isoform switching as a mechanism of acquired resistance to mutant Isocitrate dehydrogenase inhibition. Cancer Discov. 2018;8(12):1540-7.

90. Wei H, Wang Y, Zhou C, Lin D, Liu B, Liu K, et al. Distinct genetic alteration profiles of acute myeloid leukemia between Caucasian and eastern Asian population. J Hematol Oncol. 2018;11(1):18.

\section{Publisher's Note}

Springer Nature remains neutral with regard to jurisdictional claims in published maps and institutional affiliations.

Ready to submit your research? Choose BMC and benefit from:

- fast, convenient online submission

- thorough peer review by experienced researchers in your field

- rapid publication on acceptance

- support for research data, including large and complex data types

- gold Open Access which fosters wider collaboration and increased citations

- maximum visibility for your research: over $100 \mathrm{M}$ website views per year

At $\mathrm{BMC}$, research is always in progress.

Learn more biomedcentral.com/submission 